\title{
Small Interfering RNAs Induce Target-Independent Inhibition of Tumor Growth and Vasculature Remodeling in a Mouse Model of Hepatocellular Carcinoma
}

Mathieu Bergé, ${ }^{* \dagger}$ Philippe Bonnin, ${ }^{\dagger \neq}$ Eric Sulpice, ${ }^{, \dagger}$ José Vilar, ${ }^{\S \pi}$ David Allanic, ${ }^{, \dagger}$ Jean-Sébastien Silvestre, ${ }^{\S}$ Bernard I. Lévy, Gordon C. Tucker, ${ }^{\|}$Gérard Tobelem, ${ }^{\star \dagger \ddagger}$ and Tatyana Merkulova-Rainon* ${ }^{\star}$

From the Institut des Vaisseaux et du Sang," INSERM U965 Equipe Labellisée LIGUE 2009, ${ }^{\dagger}$ AP-HP Hôpital Lariboisière, ${ }^{\ddagger}$ Université Paris 7, Paris; INSERM U970, "Université Paris 5, "I Paris; and Institut de Recherches SERVIER, Cancer Research and Drug Discovery," Croissy-sur-Seine, France

RNA interference mediated by small interfering RNAs (siRNAs) has emerged as a potential therapeutic approach to treat various diseases, including cancer. Recent studies with several animal models of posttraumatic revascularization demonstrated that synthetic siRNAs may produce therapeutic effects in a target-independent manner through the stimulation of the toll-like receptor-3 (TLR3)/interferon pathway and suppression of angiogenesis. To analyze the impact of siRNAs on tumor angiogenesis, we injected transgenic mice developing hepatocellular carcinoma (HCC) with either control siRNAs or siRNA targeting neuropilin-1. We found that treatment with these siRNAs led to a comparable reduction in tumor liver volume and to inhibition of tumor vasculature remodeling. We further determined that TLR3, which recognizes double-stranded siRNA, was up-regulated in mouse HCC. Treatment of HCC mice with polyinosinic-polycytidylic acid [poly(I:C)], a TLR3 agonist, led to both a reduction of tumor liver enlargement and a decrease in hepatic arterial blood flow, indicating that TLR3 is functional and may mediate both anti-angiogenic and anti-tumor responses. We also demonstrated that siRNAs increased interferon- $\gamma$ levels in the liver. In vitro, interferon- $\gamma$ inhibited proliferation of endothelial cells. In addition, we found that siRNAs inhibited endothelial cell proliferation and morphogenesis in an interferon- $\gamma$-independent man- ner. Our results suggest that synthetic siRNAs inhibit target-independently HCC growth and angiogenesis through the activation of the innate interferon response and by directly inhibiting endothelial cell function. (Am J Pathol 2010, 177:3192-3201; DOI: 10.2353/ajpath.2010.100157)

RNA interference (RNAi) is a naturally occurring mechanism of sequence-specific gene expression silencing that is mediated by double-stranded RNA and has been implicated in the control of the expression of endogenous and exogenous genes, including those of viruses and transposons. ${ }^{1}$ RNAi, induced by artificial synthetic small interfering RNA (siRNAs) or plasmid or viral vectors encoding for short hairpin RNAs, has become a powerful tool to analyze gene function and has emerged as a potential therapeutic strategy to treat various pathologies including cancers. ${ }^{2}$ Numerous siRNAs and short hairpin RNAs have been tested in preclinical cancer models and have provided encouraging results including an efficient inhibition of tumor angiogenesis, tumor cell proliferation and invasiveness, promotion of apoptosis, uphold of differentiation, and sensitization to chemotherapy. ${ }^{3}$ Several RNAi-based approaches have recently entered clinical trials. ${ }^{4}$

Hepatocellular carcinoma (HCC) is the sixth most common cancer worldwide, with a continuously increasing incidence, and is the third cause of cancer-related deaths. ${ }^{5}$ The principal treatment modalities for HCC include surgical rejection, liver transplantation, and local ablation, whereas HCC is relatively resistant to systemic

\footnotetext{
Supported in part by research funding from Servier Laboratories.

Accepted for publication August 30, 2010.

Supplemental material for this article can be found on http://ajp. amjpathol.org

Address reprint requests to Tatyana Merkulova-Rainon, Ph.D., Institut des Vaisseaux et du Sang/INSERM U965, Hôpital Lariboisière, 8 rue Guy Patin, 75475 Paris Cedex 10, France. E-mail: tatiana.merkoulova@inserm.fr.
} 
therapy. ${ }^{6}$ RNAi-based approaches are currently being considered as potential new therapeutics to both prevent $\mathrm{HCC}$ by treating the underlying liver diseases and to cure established $\mathrm{HCC}^{7}$ In animal models of HCC or of precancerous liver diseases, synthetic siRNA-mediated gene silencing targeting hepatitis $B^{8}$ or hepatitis $C^{9}$ viral RNAs and replication intermediates or endogenous liver proteins including the Fas receptor, ${ }^{10}$ vascular endothelial growth factor-A (VEGF-A), ${ }^{11,12}$ cell-cycle-activating phosphatase $\mathrm{CDC} 25 \mathrm{~B},{ }^{13}$ and heme oxygenase $1,{ }^{14}$ has been shown to efficiently reduce target protein expression and inhibit disease progression. Another promising RNAi-based therapeutic strategy consists in the administration of microRNAs, which are down-regulated in HCC. ${ }^{15}$ Such a treatment has been found to effectively inhibit the proliferation of cancer cells and stimulate apoptosis and to strongly reduce liver tumor growth without inducing deleterious toxic effects.

$\mathrm{HCC}$ is a highly vascularized tumor, and its progression and prognosis is associated with angiogenesis and vascular remodeling. ${ }^{16}$ Arterialization of the blood supply is one of the main features of HCC and a part of the noninvasive criteria to establish an HCC diagnosis. ${ }^{16-18}$ Other vascular changes in HCC include increase in microvessel density, formation of immature vessels with increased permeability, sinusoidal capillarization, and development of intrahepatic shunts. ${ }^{16,18}$ Several studies have demonstrated that VEGF-A, a key regulator of vessel growth and homeostasis, is overexpressed in HCC and plays a central role in vascular remodeling, angiogenesis and HCC progression. ${ }^{18,19}$ Targeting angiogenesis by use of RNAi-based strategies directed against different components of VEGF signaling system appears as a rational strategy for therapy of HCC.

We have recently described the main steps of angiogenesis and vascular remodeling in a transgenic mouse model of $\mathrm{HCC} .{ }^{20}$ The evolution of $\mathrm{HCC}$ in this model is reproducible and characterized by a classical sequence of events: hyperplasia, dysplasia (between the fourth and eighth week after birth), adenoma (from the 12th week), and carcinoma (at the 16th week). As in humans, angiogenesis precedes then accompanies hepatocarcinogenesis in this model, and the VEGF-Delta-like 4/Notch4ephrin B2 pathway plays a major role in the regulation of tumor vessel growth and remodeling and HCC progression. ${ }^{21}$ We demonstrated that the hepatocellular tumor growth and vascular remodeling can be followed noninvasively in this model by using ultrasound Doppler imaging. ${ }^{22}$ We used this model to evaluate the therapeutic potential of targeting angiogenesis in HCC by treatment the mice with synthetic siRNA specific for neuropilin-1 (NRP1), a nontyrosine kinase receptor for VEGF. Moreover, it has been shown recently that synthetic siRNAs inhibit angiogenesis in a sequence- and a target-independent manner in the mouse models of choroidal neovascularization, dermal wound healing, ${ }^{23}$ corneal neovascularization, and hindlimb ischemia. ${ }^{24}$ Therefore we treated HCC mice with two different control siRNAs to analyze whether tumor angiogenesis could be targetindependently suppressed by siRNAs.

\section{Materials and Methods}

\section{Mouse Transgenic Model of HCC}

Approval was obtained from the local ethical committee for studies involving mice. The generation of ASV-B transgenic mice (C57BL/6 background) as a model of HCC has been reported previously. ${ }^{25}$ Because of the integration of the transgene into chromosome $\mathrm{Y}$, only male mice develop HCC. The female mice of the same lineage do not harbor the transgene and were used as wild-type controls. The absence of structural and functional differences in liver architecture and liver vasculature between wild-type C57BL/6 male and female mice has been demonstrated previously. ${ }^{20}$

\section{siRNA Treatment, Microangiography, and Ultrasound Study}

Silencer In vivo Ready Predesigned siRNA targeting mouse NRP1 (NRP1 siRNA) and nontargeting Negative Control \# 1 siRNA (NT siRNA) were purchased from Ambion (ID \# 155679 and 103860, respectively, Austin, TX). siSTABLE siRNA targeting the firefly enzyme luciferase (Luc siRNA, sense $5^{\prime}$-UAAGGCUAUGAAGAGAUACdTdT- ${ }^{\prime}$ and antisense $5^{\prime}$-GUAUCUCUUCAUAGCCUUAdTdT-3') ${ }^{23,24}$ was purchased from Dharmacon (Lafayette, CO). siRNAs were resuspended in sterile phosphate buffered saline (PBS) and injected intraperitoneally, twice a week, at $1.2 \mathrm{mg} / \mathrm{kg}$ into both wild-type and HCC mice. Polyinosinic-polycytidylic acid [poly(I:C)] was purchased from Sigma-Aldrich (St. Louis, MO). For injection, poly $(\mathrm{I}: \mathrm{C})$ was resuspended in PBS and administered intraperitoneally, twice a week, at $2 \mathrm{mg} / \mathrm{kg}$.

Treatments were started when mice reached the age of 8 weeks and continued until mice were 16 weeks old. Ultrasound imaging was performed every 2 weeks. Echoderived liver volume and time-average mean blood flow velocities (BFV) were estimated using a Vivid 7 echocardiograph with a 12-MHz linear transducer (GE Medical Systems ultrasound, Horten, Norway) as previously described. ${ }^{22}$ At the end of treatments, a subgroup of mice was subjected to microangiographic analysis of liver vascularization. Microangiography was performed as previously described with minor modifications. ${ }^{22}$ Before perfusion with barium sulfate $(1 \mathrm{~g} / \mathrm{ml})$, the mesenteric artery and aorta upstream from the celiac trunk were ligated. Image acquisition was performed with a digital Trophy X-ray transducer (Trophy Radiologie, Vincennes, France). Quantification of arterial vessel density was performed using the PRIMed Angio v0.9 software (Primed Microvision, France) and expressed as a percentage of pixels per image occupied by vessels in the total liver area. Another subgroup of treated mice was sacrificed, livers were excised, weighed, and processed for RNA and protein extraction and immunohistochemical analysis. 


\section{Cell Culture}

All cell culture reagents were purchased from Invitrogen (Cergy Pontoise, France) unless otherwise specified.

Human umbilical vein endothelial cells (HUVECs) were isolated and cultured as previously described. ${ }^{26}$ Lungderived normal human microvascular endothelial cells (HMVECs) were purchased from Lonza (Walkersville, $\mathrm{MD}$ ) and cultured according to the manufacturer's instructions.

The human HCC cell lines Hep G2, SK-HEP-1, and PLC/PRF/5 were purchased from the American Type Culture Collection (Rockville, MD). Cells were cultured in RPMI 1640 (Hep G2) or DMEM (SK-HEP-1 and PLC/ PRF/5) basal medium supplemented with $10 \%$ fetal bovine serum (FBS, BioWest, Cholet, France), $50 \mathrm{U} / \mathrm{ml}$ penicillin, $50 \mu \mathrm{g} / \mathrm{ml}$ streptomycin, and $2.5 \mu \mathrm{g} / \mathrm{ml} \mathrm{am}$ photericin $\mathrm{B}$.

Peripheral blood mononuclear cells (PBMCs) were isolated from blood of healthy donors by density gradient centrifugation using Pancoll (Pan-Biotech GmbH, Aidenbach, Germany). Cells were washed twice with PBS, resuspended in RPMI 1640 medium supplemented with $5 \%$ FBS at $3 \times 10^{6}$ cells $/ \mathrm{ml}$, and incubated in 24-well plates $(0.5 \mathrm{ml} / \mathrm{well})$ in the presence or absence of 50 $\mu \mathrm{g} / \mathrm{ml}$ of poly(l:C) or NT siRNA. After 24 hours, supernatants were harvested, centrifuged at $14000 \times g$ for 10 minutes, and interferon- $\gamma($ INF- $\gamma$ ) levels were determined by ELISA.

\section{Antibodies, Western Blotting, and Immunofluorescence Analysis}

The rat anti-CD31 antibody was purchased from BD Pharmingen (San Diego, CA). The goat anti-neuropilin-1 antibody was purchased from R\&D Systems (Minneapolis, MN). The rabbit anti-laminin antibody was purchased from Sigma-Aldrich (Saint Louis, MO). The rabbit antiKi-67 antibody was purchased from NeoMarkers (Fremont, CA). The goat anti-TLR3 and rabbit anti-tubulin antibody were purchased from Santa Cruz Biotechnology (Santa Cruz, CA). The rabbit anti-phospho-TLR3 (Tyr 759) antibody was purchased from Imgenex (San Diego, CA). The rabbit anti-phospho-IRF-3 (Ser 396), anti-phospho-NF-кB p65 (Ser 536) and the mouse anti-caspase-8 antibody were purchased from Cell Signaling Technology (Danvers, MA). Alexa Fluor 488-conjugated donkey antirat lgG, Alexa Fluor 555-conjugated donkey anti-goat lgG and Alexa Fluor 555-conjugated goat anti-rabbit antibody were purchased from Molecular Probes (Eugene, OR). Peroxidase-conjugated species-specific secondary IgGs were obtained from Jackson ImmunoResearch (West Grove, PA).

Liver samples were homogenized in RIPA buffer using a MagNA Lyser Instrument (Roche, Rotkreuz, Switzerland). Protein extraction, Western blotting, band visualization, and quantification were performed as previously described. ${ }^{27}$

For immunofluorescence analysis, frozen liver sections $(5 \mu \mathrm{m})$ or cell cultures were fixed in cold acetone (15 minutes), washed three times with PBS containing $0.1 \%$
Tween-20 (PBST), and incubated in PBST containing $0.1 \%$ BSA for 20 minutes. Samples were then incubated with primary antibody for 1 hour at room temperature followed by the appropriate secondary antibody (30 minutes). Negative controls were incubated with the secondary antibodies only. Sections were observed with an Axioskop 2 plus fluorescence microscope equipped with an Achroplan $\times 40 / 0.65$ and a Plan-Neofluar $\times 5 / 0.16$ Ph1 objectives (Carl Zeiss Microlmaging GmbH, Göttingen, Germany). Images were taken using a QICAM 12 bit Fast 1394 Cooled Color Camera (QImaging, Pleasanton, CA) and Archimed 5.6 software (Microvision Instruments, Evry, France), and quantified using Histolab 5.8 software (Microvision Instruments). Cell cultures were observed using an Observer.Z1 inverted microscope equipped with an EC Plan-Neofluar $\times 40 / 0.75$ objective (Carl Zeiss). Images were taken with a KY-F75U digital camera (JVC, Tokyo, Japan).

\section{Real-Time RT-PCR}

Total cellular RNA was extracted with RNAXEL reagent (Eurobio, Les Ulis, France) and purified using the RNeasy Midi Kit (Qiagen, Hilden, Germany) according to the manufacturer's instructions. The first strand cDNA template was synthesized using the Transcriptor First Strand cDNA Synthesis Kit and random hexamer primers (Roche Applied Science, Mannheim, Germany). The cDNA product was amplified using LightCycler FastStart DNA Master SYBR Green I and a Light Cycler 1.5 (Roche). The primers used for PCR were: mouse TLR3 forward 5'TTGTCTTCTGCACGAACCTG-3', and mouse TLR3 reverse 5'-CGCAACGCAAGGATTTTATT-3'; mouse 18S forward 5'-CGCGGTTCTATTTTGTTGGT-3', and mouse $18 \mathrm{~S}$ reverse 5'-AGTCGGCATCGTTTATGGTC-3'.

mRNA levels were normalized to the $18 \mathrm{~S}$ ribosomal RNA. TLR3 mRNA was detected in human cell lines after cDNA amplification with FastStart TaqDNA Polymerase (Roche) and following primers: human TLR3 forward 5' AGCCTTCAACGACTGATGCT-3', and human TLR3 reverse 5'-TTTCCAGAGCCGTGCTAAGT-3'.

\section{ELISA}

Interferon- $\alpha,-\beta$, and interleukin-12 concentrations in liver homogenates were estimated using the Mouse Interferon $\alpha$ ELISA Kit, Mouse Interferon $\beta$ ELISA Kit, and Quantikine Mouse IL-12 p70 Immunoassay (R\&D Systems), respectively, according to the manufacturer's instructions. The BD OptEIA mouse IFN- $\gamma$ ELISA Set (BD Biosciences, San Diego, CA) was used to quantify interferon- $\gamma$. Data were normalized to total protein content estimated with the BCA Protein Assay Reagent (Pierce, Rockford, IL). Interferon- $\gamma$ concentration in human PBMC supernatants was determined using Quantikine Human INF- $\gamma$ Immunoassay (R\&D Systems).

\section{MTT Assay}

Cells were plated in 96-well plates at a density of $10^{4}$ cells per well (HUVEC and Hep G2) or $5 \times 10^{3}$ cells per 
well (SK-HEP-1 and PLC/PRF/5) and allowed to grow at $37^{\circ} \mathrm{C}$ with $5 \% \mathrm{CO}_{2}$ for 48 hours. Cells were then incubated with various concentrations of INF- $\gamma$ (Reliatech GmBH, Wolfenbüttel, Germany), poly(l:C), NRP1 siRNA or NT siRNA for a further 48 hours, followed by an incubation with $0.5 \mathrm{mg} / \mathrm{ml}$ of 3-(4,5-dimethyl-2-thiazolyl)-2,5diphenyl-2H-tetrazolium bromide (MTT, Sigma-Aldrich). After 4 hours, a water-insoluble formazan produced by viable cells was solubilized with DMSO and cell viability and growth over time was estimated by measuring the absorbance at $560 \mathrm{~nm}$ using a Victor3 spectrofluorimeter (Perkin Elmer, Turku, Finland).

\section{Three-Dimensional Collagen Assay}

HUVECs were resuspended in M199 medium supplemented with $1 \%$ FBS and seeded at $10^{5}$ cells per well on the top of a preformed collagen gel (BD Biosciences, Bedford, MA) in 24-well plate. Capillary-like tube formation was induced by the addition of $100 \mathrm{ng} / \mathrm{ml}$ of VEGF$A_{165}$ and HGF (R\&D systems) in the presence or absence of $100 \mathrm{ng} / \mathrm{ml}$ of INF- $\gamma, 50 \mu \mathrm{g} / \mathrm{ml}$ of poly(l:C), or $50 \mu \mathrm{g} / \mathrm{ml}$ of NT siRNA. After 24 hours of incubation, cultures were observed using an Observer.Z1 inverted microscope equipped with an EC Plan-Neofluar 10x/0.3 Ph1 objective (Carl Zeiss). Images were taken with a KY-F75U digital camera (JVC) and quantified using Histolab 5.8 software.

\section{Statistical Analysis}

Data are presented as the mean \pm SEM. The echoderived volume of the liver, heart rate, peak systolic, end-diastolic, and mean BFV in hepatic and mesenteric arteries were compared between HCC mice treated with PBS, NRP1 siRNA, or control siRNAs by analysis of variance for repeated measurements (with time as within subject factor and group as between subject factor) followed by post hoc Student's t-test with Bonferroni correction (MedCalc Software, Mariakerke, Belgium). Statistical analyses of other data were performed in Excel using Student's $t$-test. Data were considered statistically significant if $P<0.05$.

\section{Results}

\section{Treatment with Either NRP1 or Control siRNAs Inhibits Tumor Growth in a Mouse Model of Hepatocellular Carcinoma}

To evaluate the effect of synthetic siRNAs on HCC growth and angiogenesis, we used an siRNA targeting the VEGF receptor NRP1 (NRP1 siRNA) and two control siRNAs, one non-targeting (NT siRNA), with limited sequence similarity to known mouse, human, and rat genes, and the other one targeting the firefly enzyme luciferase (Luc siRNA). The NRP1 siRNA was selected from preliminary in vitro experiments based on its capacity to effectively down-regulate (by 90\%) NRP1 protein expression in a cultured mouse melanoma cell line B16-F10, which ex- presses high levels of NRP1 (data not shown). We found that twice a week intraperitoneal administration of this siRNA (at $1.2 \mathrm{mg} / \mathrm{kg}$ ) reduced the expression of NRP1 protein by $25 \%$ in the liver of transgenic mice developing HCC (data not shown). In contrast, the control siRNAs, which had no effect on NRP1 expression in B16-F10 cells, did not significantly modulate the NRP1 protein level in the liver.

We have previously established that between the fourth and 20th weeks, hepatocellular tumor growth is accompanied by an approximately sixfold increase in liver volume that can be assessed using ultrasound imaging. ${ }^{22}$ We used this approach to follow the rate of HCC growth in siRNA-treated mice. Ultrasonography demonstrated that similar to our previous observations, the echo-derived liver volume was increased almost threefold between the eighth and 16th week in HCC mice treated with vehicle alone (Figure 1A). Treatment with NRP1 siRNA inhibited liver volume growth in HCC mice (1.9-fold increase, $P=0.0053$ versus PBS), whereas it did not affect liver volume in wild-type mice. The two control siRNAs decreased HCC growth to an extent similar to that observed with the NRP1 siRNA (Figure 1A). These results were confirmed by weighing the livers after sacrifice at the 16th week (Figure 1B).

We showed previously that capillarization of blood supply in mouse HCC is accompanied by the up-regulation of laminin expression within tumor nodules. ${ }^{20} \mathrm{We}$ used laminin immunostaining to visualize tumor nodules in liver sections of treated mice and determined their size with Histolab software. We found that treatment with each siRNA led to the reduction of tumor nodule size compared to PBS-treated animals (Figure 1C). Whereas the number of tumor nodules per section was higher in siRNA-treated mice $(4.5 \pm 0.75,2.67 \pm 0.82,4.75 \pm$ 0.87, for NRP1 siRNA, NT siRNA, and Lus NRP1 siRNA, respectively, against 1 nodule per section of PBS treated mice) the percentage of section area occupied by all tumor nodules was significantly lower in siRNA-treated mice compared to PBS-treated mice (Figure 1C). Thus, our results suggest that treatment with siRNAs does not affect the incidence of tumor nodules but limit their growth.

Finally, to verify whether the decrease in HCC liver growth correlated with a decrease in tumor cell proliferation, we costained liver sections from sacrificed animals with 4'-6-diamidino-2-phenylindole (DAPI) and the proliferation marker Ki-67. We found that the proportion of Ki-67-positive nuclei was reduced by $30-40 \%$ in the liver of HCC mice treated with siRNAs compared to mice treated with PBS (Figure 1D). Thus, the siRNA-induced inhibition of HCC growth could at least be partially explained by the inhibition of proliferation of tumor hepatocytes.

\section{siRNA Treatment Inhibits Angiogenesis and Vascular Remodeling in a Mouse Model of Hepatocellular Carcinoma}

We next analyzed whether treatment with synthetic siRNAs affected tumor vasculature in transgenic mice develop- 
A

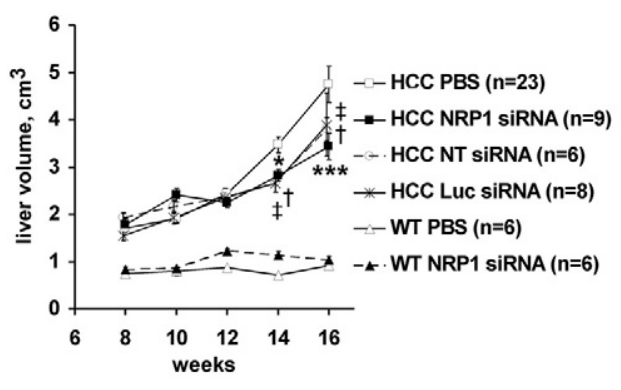

B

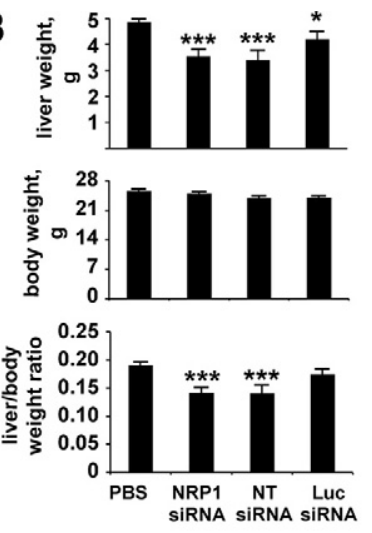

C
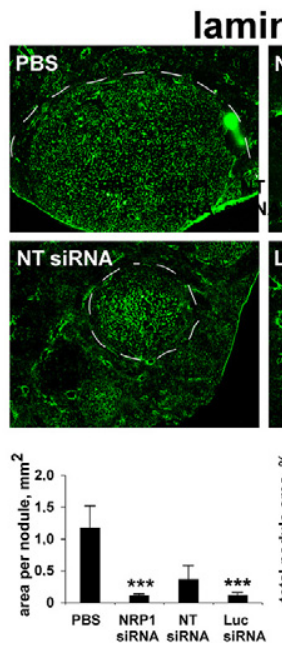

laminin
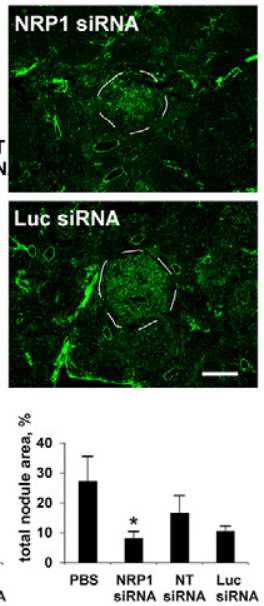

D
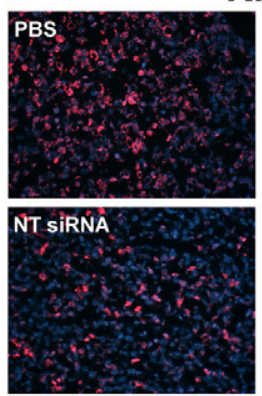

Ki-67
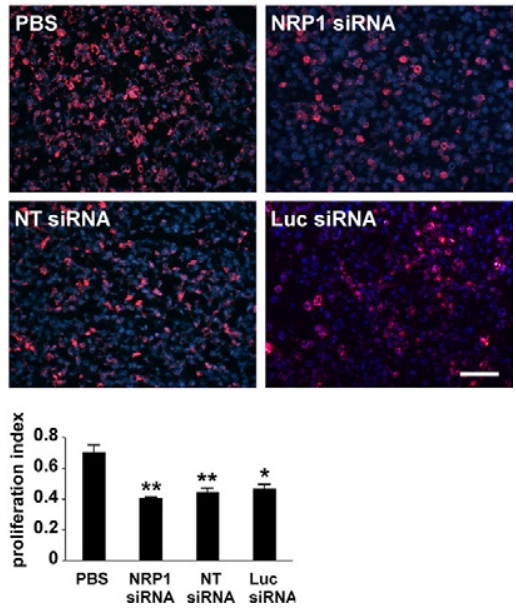

Figure 1. siRNA-induced inhibition of mouse hepatocellular carcinoma growth is target-independent. A: The volume of the liver of treated mice was estimated using two-dimensional ultrasound imaging. Both NRP1 and control siRNAs significantly delayed the increase in tumor liver volume compared to PBS-treated mice. ${ }^{*} P<$ 0.05 (NRP1 siRNA versus PBS); ${ }^{* * * *} P<0.001$ (NRP1 siRNA versus PBS); ${ }^{\dagger} P<0.05$ (NT siRNA versus PBS); ${ }^{\ddagger} P<0.05$ (Luc siRNA versus PBS) (Student's $t$-test). B: Liver weight, body weight, and liver/body weight ratio of treated mice at 16 weeks. Treatment with either NRP1 or control siRNAs led to a reduction of tumor liver weight ${ }^{*} P<0.05 ;{ }^{* * * *} P<0.001$ (Student's $t$-test). C: Sections of livers of treated mice harvested at 16 weeks were stained with anti-laminin antibody to visualize tumor nodules. Nodules size (four mice per treatment group) was quantified using Histolab 5.8 software (Microvision Instruments). Representative micrographs are shown together with quantification results expressed as area per individual nodule and the percentage of section area occupied by tumor nodules. Scale bar, 400 $\mu \mathrm{m} .{ }^{*} P<0.05$; ${ }^{* * * *} P<0.001$ (Student's $t$-test). D Sections of livers harvested at 16 weeks were double-stained for Ki-67, a proliferation marker (red), and the nuclear marker DAPI (blue). Ki67-positive nuclei were counted in seven randomly selected microscopic fields in sections of three different liver samples per treatment group. Representative micrographs are shown together with quantification results expressed as a ratio of Ki-67-positive nuclei to total number of nuclei. Less Ki-67-positive nuclei were detected in livers of mice treated with siRNAs. Scale bar, $50 \mu \mathrm{m} .{ }^{*} P<0.05$; ${ }^{* *} P<0.01$ (Student's $t$-test). ing HCC. We have previously demonstrated that as in humans, HCC progression in a mouse transgenic model is accompanied by a strong remodeling of tumor sinusoids, sinusoidal capillarization, and arterialization of the blood supply. ${ }^{20,22}$ These events led to an increase in hepatic artery BFV that could be measured using Doppler ultrasound imaging. As expected, Doppler ultrasonography demonstrated that hepatic artery mean BFV was significantly higher in HCC mice compared with wild-type mice, and it increased progressively from the eighth to the 16th week (Figure 2A). Treatment with NRP1 siRNA, but also with control siRNAs, counteracted this effect [Figure 2A, $P$ (versus PBS) of $0.002,0.035$ and 0.003, for NRP1 siRNA, NT siRNA and Luc siRNA, respectively], whereas none of the siRNAs affected hepatic artery BFV in wild-type mice (Figure 2A) or mesenteric artery BFV in both wild-type and HCC mice (see Supplemental Figure S1 at http://ajp.amjpathol.org). As evident by microangiography, treatment with the NRP1 and control siRNAs reduced the hepatic arterial vascularization (by $30 \%$ and $20 \%$, respectively; Figure $2 \mathrm{~B}$ ), which is dramatically increased in HCC mice compared to wildtype mice. ${ }^{22}$ In contrast, renal vascularization was not modified in treated animals, suggesting specificity of siRNA-induced effects regarding tumor vasculature. Finally, using immunohistochemical analysis we demonstrated that the abnormal sinusoidal architecture characterized by the formation of irregular, dilated, and fusing hepatic sinusoids was partially normalized in HCC mice treated with NRP1 siRNA and to a lesser extent in mice treated with control siRNAs (Figure 2C).

\section{siRNA Treatment Induces an Innate Interferon Response in HCC Mice}

Inhibition of mouse HCC growth and vascular remodeling by both NRP1 and control siRNAs revealed a targetindependent mode of action and suggests the involvement of an innate interferon response stimulated by siRNA. ${ }^{23,24}$ Indeed, although we were unable to detect interferon- $\alpha$ in liver extracts of HCC mice (data not shown), we demonstrated that liver production of interferon- $\gamma($ INF- $\gamma)$ was significantly increased in HCC mice after treatment with either NRP1 or control siRNAs, whereas interferon- $\beta$ and interleukin-12 levels were not statistically significantly modified (Figure 3).

Taking into account the recent findings on the role of toll-like receptor 3 (TLR3) in siRNA-mediated vascular effects, ${ }^{23,24}$ we analyzed TLR3 expression in untreated HCC mice. We found that mRNA encoding for mouse TLR3 was expressed in livers of both wild-type and HCC mice, and that it was up-regulated in HCC mice between the fourth and $16^{\text {th }}$ week (Figure $4 \mathrm{~A}$ ). Double-staining immunohistochemical experiments identified hepatic endothelial cells as the most abundant cell type expressing 
A

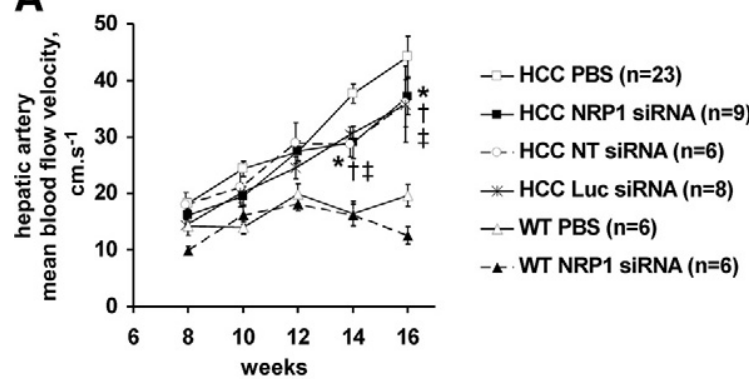

B
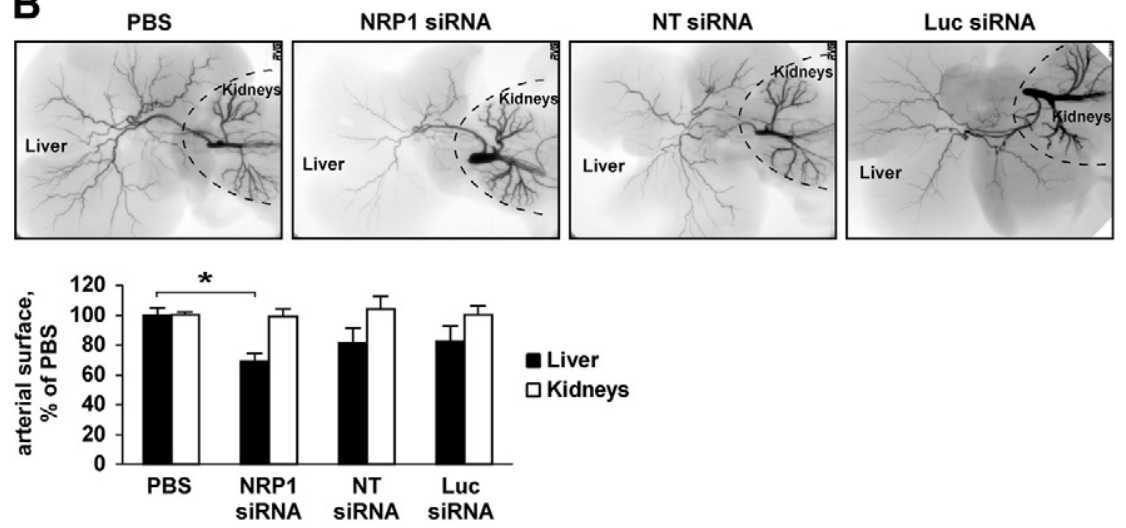

C wT

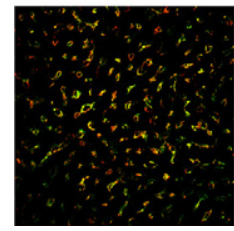

NRP1 SIRNA

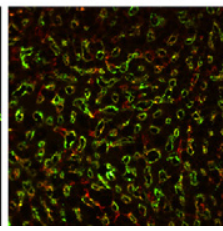

NT SIRNA

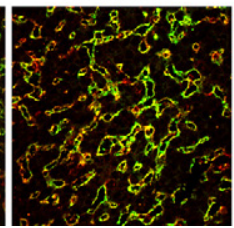

Luc siRNA

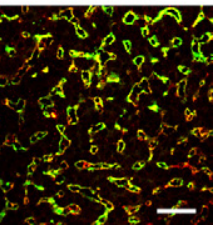

Figure 2. Both NRP1 and control siRNAs inhibit tumor angiogenesis and vascular remodeling in HCC mice. A: Time average mean BFV in the hepatic artery of treated mice was estimated using Doppler ultrasound imaging. Both NRP1 and control siRNAs significantly delayed the increase in hepatic artery BFV indicating inhibition of tumor vasculature remodeling. ${ }^{*} P<0.05$ (NRP1 siRNA versus $\mathrm{PBS}$ ); ${ }^{\dagger} P<0.05$ (NT siRNA versus PBS); ${ }^{\ddagger} P<0.05$ (Luc siRNA versus PBS) (Student's $t$-test). B: A contrast agent was injected into the aorta of treated mice and the arterial phase in the liver and kidneys was analyzed using microangiography. Arterial vessel density was quantified with a PRIMed Angio v0.9 software. As evident by microangiography, NRP1 siRNA and to a lesser extent control siRNAs inhibited arterialization of the blood supply in HCC mice. ${ }^{*} P<0.05$ (Student's $t$-test). C: CD31 (green) and NRP1 (red) double immunostaining of liver sections harvested from mice at 16 weeks. In mice treated with siRNAs, liver vascularization was characterized by more regular, less fused sinusoids that resemble those in wildtype liver (left micrograph). Scale bar, $50 \mu \mathrm{m}$.
TLR3 protein. In addition, in HCC mice, a population of tumor hepatocytes was positively stained for TLR3 (Figure $4 \mathrm{~B}$, shown at 12 weeks).

To assess the contribution of liver-expressed TLR3 to the control of HCC progression, we injected poly(l:C), a specific ligand for TLR3, into HCC mice and analyzed its effect on HCC tumor growth and angiogenesis. We found that twice a week administration of poly(l:C) at $2 \mathrm{mg} / \mathrm{kg}$ between the eighth and 16th weeks delayed tumor liver enlargement and inhibited the increase in hepatic artery BFV, compared with PBS-treated mice (Figure 4C). Thus,

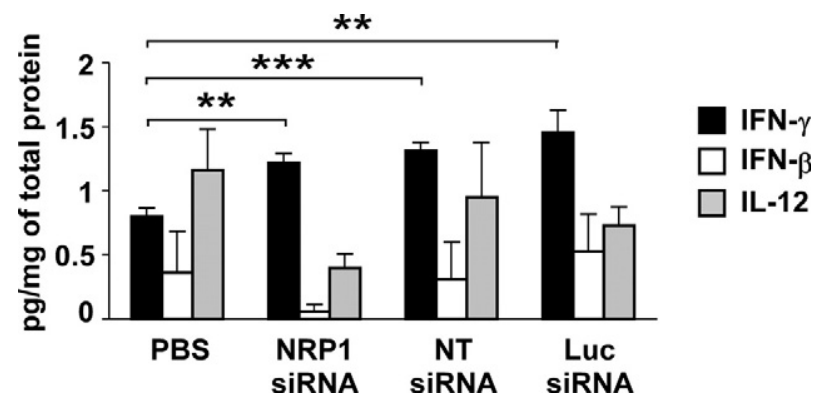

Figure 3. Interferon- $\gamma$ production is increased in the livers of siRNA-treated HCC mice. INF- $\beta,-\gamma$, and interleukin-12 levels in liver homogenates at 16 weeks were quantified using ELISA. Both NRP1 and control siRNAs significantly increase INF- $\gamma$ levels in the livers of treated mice. ${ }^{* * *} P<0.01 ;{ }^{* * * *} P<$ 0.001 (Student's $t$-test).
TLR3 is functional in hepatocellular tumors and could mediate the antiangiogenic and antitumor responses.

\section{TLR3 Ligands Inhibits Cells Functions in Vitro in an INF- $\gamma$-Independent Manner}

To explore the molecular mechanisms underlying the siRNAs and poly(I:C)-mediated inhibition of HCC growth, and possible involvement of TLR3, we analyzed the effects of poly(l:C) and siRNAs on cultured endothelial cells and on cell lines derived from hepatic tumors.

We first determined that cultured micro- and macrovascular endothelial cells (HMVECs, HUVECs) and hepatic tumor-derived cells Hep G2, SK-HEP-1, and PLC/ PRF/5 express TLR3 mRNA and are positive for TLR3 immunostaining (see Supplemental Figure S2, A and B at http://ajp.amjpathol.org). We found, however, that these cells did not secrete INF- $\gamma$, in standard culture conditions or after incubation (up to 96 hours) with poly(I:C) or siRNAs (data not shown). This was in contrast to PBMCs, a peripheral blood cell fraction enriched in immune cells, which secreted INF- $\gamma$ in response to poly(I:C) and, less efficiently, to NT siRNA (see Supplemental Figure S3 at http://ajp.amjpathol.org).

We further established that on treatment with poly(I:C) or siRNAs, TLR3-mediated signaling pathways were 
A

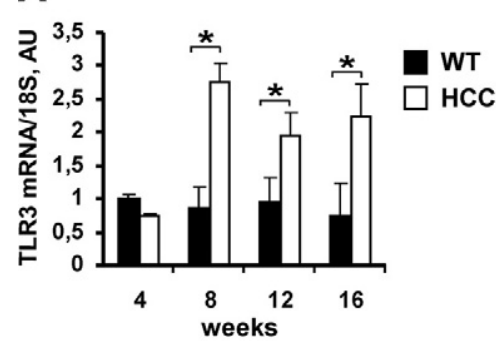

C

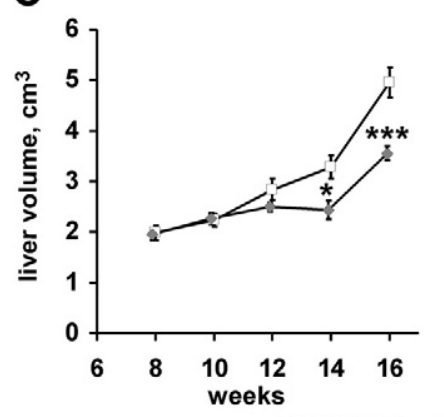

B
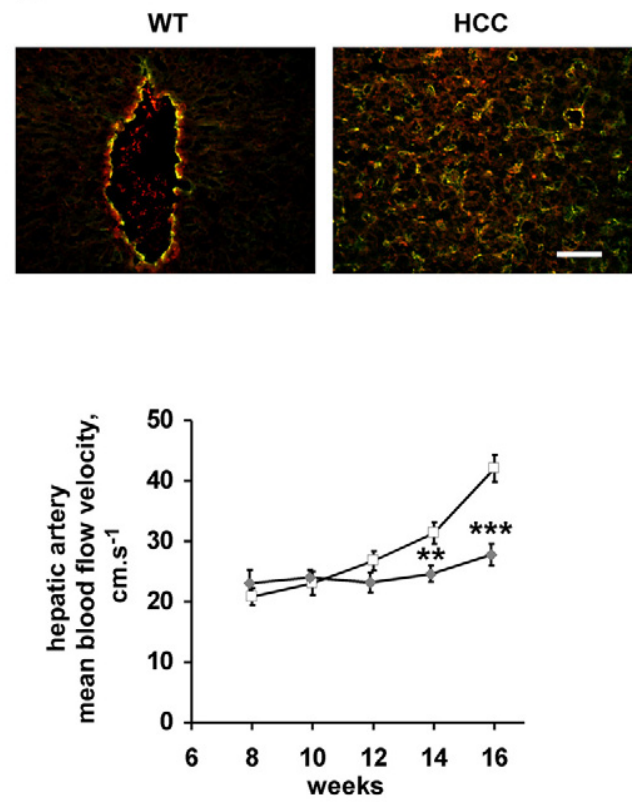

Figure 4. TLR3 is up-regulated and functional in mouse HCC. A: The amounts of TLR- 3 mRNA at different stages of postnatal development were quantified in the liver of wild-type and HCC mice using real-time RT-PCR. Data were normalized to the $18 \mathrm{~S}$ ribosomal RNA. Starting from the eighth week, TLR3 mRNA levels were up-regulated in HCC, whereas they did not vary significantly with age in the liver of wild-type mice. ${ }^{*} P<0.05$ (Student's $t$-test). B: Doublestaining with anti-CD31 (green) and anti-TLR3 (red) antibodies of liver sections from wild-type and HCC mice at 12 weeks demonstrates the localization of TLR3 in hepatic endothelial cells. In HCC mice, a population of tumor hepatocytes was positively stained for TLR3. Scale bar, 50 $\mu \mathrm{m}$. C: The volume of the liver and the hepatic artery mean BFV of mice treated with a TLR3 ligand poly(I:C) was estimated using two-dimensional ultrasound imaging and Doppler ultrasound imaging, respectively. In vivo stimulation of TLR3 decreased tumor liver enlargement and hepatic artery BFV indicating the inhibition of tumor growth and vasculature remodeling. ${ }^{*} P<$ $0.05 ;{ }^{* * *} P<0.01 ;{ }^{* * * *} P<0.001$ (Student's $t$-test).

weakly activated in HMVECs, HUVECs, Hep G2, SKHEP-1, and PLC/PRF/5 cells, as evidenced by Western blot detection of phospho-TLR3, phospho-IRF-3, phospho-NF- $\kappa \mathrm{B}$ p65, and caspase-8 cleavage (Figure 5A, shown for HUVECs and SK-HEP-1). Nevertheless, the proliferation of endothelial cells was significantly inhibited by poly(l:C) and NT siRNA, albeit this effect was less pronounced compared to INF- $\gamma$-induced inhibition (Fig-
A

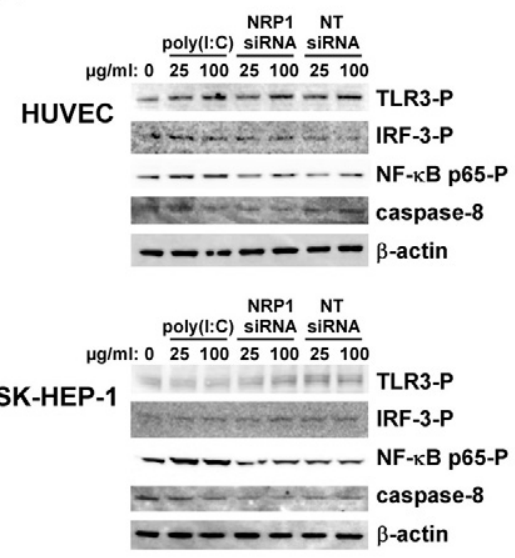

B

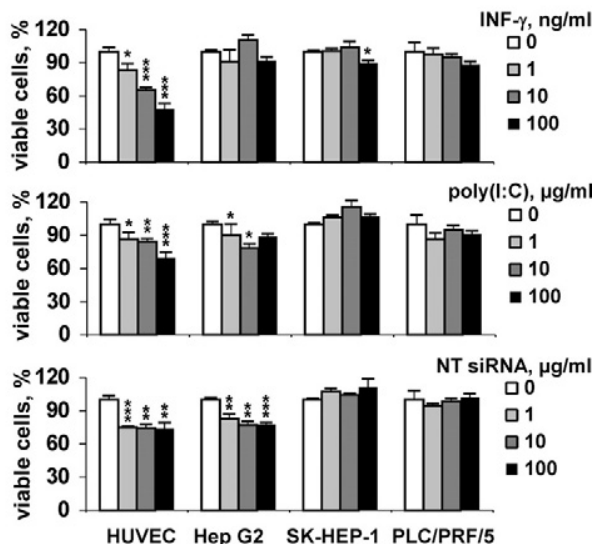

VEGF-A 165 + HGF
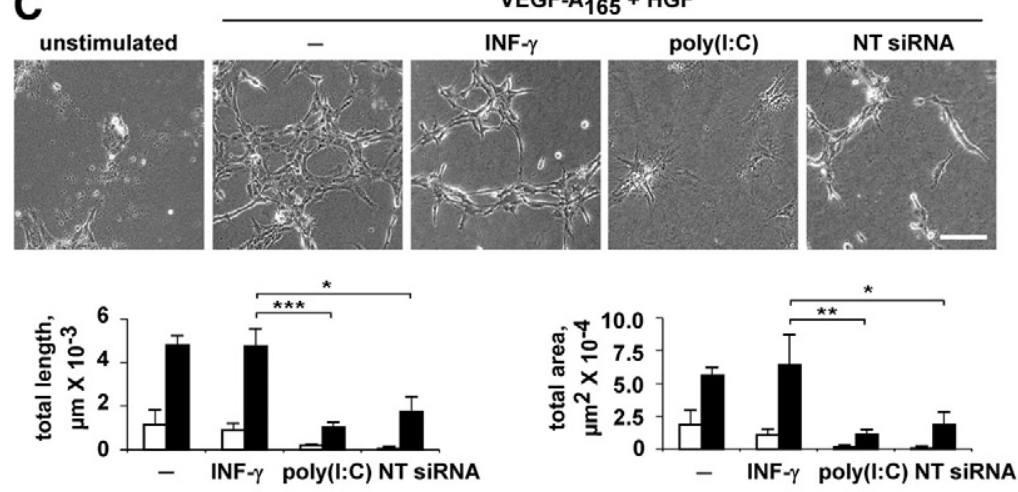

VEGF-A $165+$ HGF
Figure 5. INF- $\gamma$-independent effects of TLR3 ligands on cultured cells. A: Serum-deprived cells were incubated for 30 minutes (for phosphoprotein detection) or for 24 hours (for caspase-8 detection) with poly(I:C), NRP1 siRNA, or NT siRNA, as indicated. Cell lysates were analyzed by Western blot with phospho-site specific or anti-caspase- 8 antibodies. $\beta$-actin was used as loading control. Results shown are representative of three independent experiments. B: Cells were grown in the presence of various concentrations of INF- $\gamma$, poly(I:C), or NT siRNA for 48 hours and cell viability and growth over time was estimated using MTT assay. Results are expressed as percentage of viable cells observed in cell cultures grown in the absence of inhibitors. Data are representative of three independent experiments. C: HUVECs were cultured on collagen gels in starvation medium (M199 medium containing 1\% FBS) alone or supplemented with $100 \mathrm{ng} / \mathrm{ml} \mathrm{INF}-\gamma, 50 \mu \mathrm{g} / \mathrm{ml}$ poly(I:C), or 50 $\mu \mathrm{g} / \mathrm{ml}$ of NT siRNA. Photographs were taken at 24 hours. Scale bar, $100 \mu \mathrm{m}$. The total tube length and the tubular network area were quantified using Histolab software. Results shown are representative of four independent experiments. ${ }^{*} P<0.05 ;{ }^{* *} P<0.01 ;{ }^{* * * *} P<0.001$. Student's $t$-test). 
ure 5B). We found also that poly(l:C) and NT siRNA moderately affected proliferation of slow growing Hep G2 cell line, whereas INF- $\gamma$ was ineffective in inhibiting this cell type (Figure 5B).

Finally, we compared INF- $\gamma$, poly $(I: C)$ and siRNA activities in HUVECs cultured on 3-D collagen gel ("in vitro angiogenesis"). In this model, when cultured in low serum conditions, endothelial cells underwent rapid apoptosis (Figure 5C, unstimulated). The addition of growth factors (angiogenic cocktail containing VEGF- $\mathrm{A}_{165}$ and HGF) induces endothelial cell to adopt a spindle-shaped morphology, align into multicellular tubular-like structures and form a network similar to capillary network in vivo. We found that this process was not affected by INF- $\gamma$, as evaluated by quantification of total tubular length and tubular network area (Figure 5C). In contrast, a dramatic inhibition of tubular network formation was observed in the presence of poly $(I: C)$ and, to a lesser extent, of NT siRNA, indicating an important role of TLR3 ligands in regulating of endothelial cell morphogenesis.

\section{Discussion}

Promising prospects in systemic therapy for HCC have been initiated with the development of new antiangiogenic therapies, in particular those targeting VEGF signaling. Indeed, the recently reported phase II trial of bevacizumab, a humanized anti-VEGF monoclonal antibody, ${ }^{28}$ and sorafenib, a small tyrosine kinase inhibitor targeting VEGF receptors, ${ }^{29}$ demonstrated a survival advantage in HCC patients. However, only modest survival improvements have been demonstrated in these trials. Moreover, some patients may be refractory to these drugs or develop resistance after treatment. This highlights the need to identify new molecular targets and new therapeutic modalities to significantly improve the effectiveness of antiangiogenic therapy in HCC.

In this work we analyzed the therapeutic potential of targeting angiogenesis in HCC using synthetic siRNAs targeting or not the VEGF receptor NRP1. As therapeutic agent, synthetic siRNAs offer some advantages over the expressed short hairpin RNAs, including a dose-controlled delivery, the absence of interference with endogenous microRNA pathways and possibility for chemical modifications that could increase their efficiency and attenuate the off target suppression effects. ${ }^{30}$ Several reports have demonstrated success in inhibiting liver tumor growth in mice through the treatment with naked nonmodified synthetic siRNAs ${ }^{12,14}$ whereas some siRNA delivery formulations were found to induce the unwanted immune stimulation effects. ${ }^{31}$ We therefore decided to treat HCC transgenic mice with naked siRNAs, administered through the systemic route, under a conventional pressure, without application of hydrodynamic force.

We found that twice a week administration of NRP1 siRNA resulted in a moderate but statistically significant reduction of NRP1 protein expression in the liver of mice developing HCC. Concomitantly, HCC growth was inhibited in HCC mice treated with NRP1 siRNA. One possible mechanism underlying this effect could be delayed an- giogenesis and vascular remodeling, which ultimately leads to the inhibition of tumor progression. Indeed, we demonstrated that treatment with NRP1 siRNA decreased the hepatic artery BFV and resulted in a partially normalized vascular phenotype of hepatocellular tumors. Our data suggest, however, that therapeutic effect of NRP1 siRNA was largely target-independent because we observed a comparable inhibition of HCC vasculature remodeling and growth in mice treated with control siRNAs. Our results are consistent with recent reports on the target-independent antiangiogenic effects of a wide range of synthetic siRNAs, targeting or not VEGF-A and VEGFR-1, in several animal models of post traumatic angiogenesis. ${ }^{23,24}$ These effects were found to result from the activation of endothelial cell-surface exposed TLR3 and consequent up-regulation of INF- $\gamma$ and interleukin-12 production.

TLR3 is a member of TLR family of innate immune response receptors implicated in the initial host defense against bacteria and viruses through the recognition of specific pathogen-associated molecular patterns and stimulation of intracellular signaling leading to the secretion of inflammatory cytokines. ${ }^{32}$ TLR3 specifically recognizes double-stranded RNA from viruses, apoptotic cells, and/or necrotic cells. It is generally located in the endosomal compartments but also found on plasma membrane in several cell types, including endothelial cells. ${ }^{33}$ Earlier studies demonstrated that TLR3 is expressed in the liver, in Kupffer cells, hepatic stellate cells, hepatic immune cells, liver sinusoidal endothelial cells, and normal and tumor hepatocytes, on both their cell surface and in the cytoplasm. ${ }^{32,34,35}$ Accordingly, we found that TLR3 is expressed in the liver of transgenic HCC mice, in endothelial cells, and, starting from the adenoma stage, in a population of tumor hepatocytes. In addition, we demonstrated that TLR3 is up-regulated during HCC progression.

In agreement with the hypothesis of the involvement of TLR3 in siRNAs-mediated effects in HCC we found that treatment of HCC mice with poly(I:C), a TLR3 specific ligand, results in suppression of vasculature remodeling and tumor liver growth to an extent similar to that observed with synthetic siRNAs. However, siRNA are capable to activate the innate immune response through ligating other RNA-sensing receptors, including TLR7 and TLR8, and some cytosolic immune sensors. ${ }^{31}$ The role of these molecules in mediating antiangiogenic and antitumor effects induced by synthetic siRNA in HCC mice cannot be ruled out.

TLR3-mediated signaling leads to the activation of NF- $\kappa \mathrm{B}$ and IRF-3 pathways and expression of inflammatory genes, including interferons. ${ }^{33}$ Recent evidence indicates that TLR3 may contribute to suppression of tumor growth through the interferon-dependent activation of NK cells and expansion of Treg lymphocytes. ${ }^{36}$ We found that liver levels of INF- $\gamma$ were significantly increased in mice treated with siRNAs. Our in vitro experiments demonstrate however that siRNAs and poly $(\mathrm{l}: \mathrm{C})$ do not stimulate INF- $\gamma$ production by cultured endothelial and hepatic tumor cells. Thus, INF- $\gamma$ detected in mouse HCC liver extracts was most probably released by circulating 
or resident immune cells. Indeed, in accordance with the previously reported data, ${ }^{37,38}$ we showed that treatment of PBMCs, which are enriched in immune cells, with poly(l:C) and, to a lesser extent, with siRNA contributes to INF- $\gamma$ production by these cells.

INF- $\gamma$ has been identified as an inhibitor of endothelial cell proliferation and a potent suppressor of tumor-associated neovascularization. ${ }^{39-42}$ We showed that INF- $\gamma$ inhibits efficiently endothelial cell proliferation in vitro, whereas it has no effect on proliferation of hepatic tumor cells. Thus, the increased INF- $\gamma$ production that might be attributed to the siRNA-dependent activation of TLR3, could mediate target-independent antiangiogenic effects of siRNAs in HCC and thus lead to the inhibition of tumor growth. In addition, the direct effects of double-stranded RNA on endothelial cells, including TLR3 activation, ${ }^{24}$ interleukin-6 release and permeability changes, ${ }^{43}$ upregulation of tissue factor and down-regulation of thrombomodulin expression, ${ }^{44}$ have been reported. Some of these effects were found to occur independently of TLR3 activation. ${ }^{43}$ We demonstrate that poly $(\mathrm{I}: \mathrm{C})$ and synthetic siRNAs stimulate weakly TLR3-mediated signaling in cultured endothelial and hepatic tumor cells, whereas they inhibit endothelial cell proliferation and had minimal if any effect on hepatic cell lines. Most importantly, we found that poly $(\mathrm{I}: \mathrm{C})$ and siRNA are potent inhibitors of endothelial cell morphogenesis, a process by which the endothelial cells assemble into capillary-like structures. Interestingly, INF- $\gamma$ did not influence this endothelial cell activity. Our results demonstrate therefore that INF- $\gamma$ and TLR3 ligands affect different aspects of angiogenic response.

In conclusion, our data indicate that using synthetic siRNAs as therapeutic agent to treat HCC could result in a target-independent inhibition of tumor angiogenesis and growth through the activation of the innate interferon response and by directly inhibiting the endothelial cell function. This feature may represent a useful approach for adjuvant therapy. However, the undesirable deleterious effects, including apoptosis, fibrosis, and multiple liver failure, may occur in the case of uncontrolled interferon production. A better understanding of the targetspecific and immunostimulatory potential of each candidate siRNA and of the contribution of both to therapeutic effect is required for future development of siRNA-based treatments in HCC.

\section{Acknowledgment}

We thank Dr. John A. Hickman (Servier) for his interest in this work and helpful discussions.

\section{References}

1. Siomi $\mathrm{H}$, Siomi MC: On the road to reading the RNA-interference code. Nature 2009, 457:396-404

2. Kim DH, Rossi JJ: Strategies for silencing human disease using RNA interference. Nat Rev Genet 2007, 8:173-184

3. Huang C, Li M, Chen C, Yao Q: Small interfering RNA therapy in cancer: mechanism, potential targets, and clinical applications. Expert Opin Ther Targets 2008, 12:637-645
4. Castanotto D, Rossi JJ: The promises and pitfalls of RNA-interference-based therapeutics. Nature 2009, 457:426-433

5. Llovet JM, Bruix J: Novel advancements in the management of hepatocellular carcinoma in 2008. J Hepatol 2008, 48 Suppl 1:S20-S37

6. Yau T, Chan P, Epstein R, Poon RT: Evolution of systemic therapy of advanced hepatocellular carcinoma. World J Gastroenterol 2008, 14:6437-6441

7. Arbuthnot P, Thompson LJ: Harnessing the RNA interference pathway to advance treatment and prevention of hepatocellular carcinoma. World J Gastroenterol 2008, 14:1670-1681

8. Chen Y, Cheng G, Mahato RI: RNAi for treating hepatitis B viral infection. Pharm Res 2008, 25:72-86

9. Watanabe T, Umehara T, Kohara M: Therapeutic application of RNA interference for hepatitis C virus. Adv Drug Deliv Rev 2007, 59: 1263-1276

10. Song E, Lee SK, Wang J, Ince N, Ouyang N, Min J, Chen J, Shankar $P$, Lieberman J: RNA interference targeting Fas protects mice from fulminant hepatitis. Nat Med 2003, 9:347-351

11. Kornek M, Lukacs-Kornek V, Limmer A, Raskopf E, Becker U, Klockner M, Sauerbruch T, Schmitz V: 1,2-dioleoyl-3-trimethylammonium-propane (DOTAP)-formulated, immune-stimulatory vascular endothelial growth factor a small interfering RNA (siRNA) increases antitumoral efficacy in murine orthotopic hepatocellular carcinoma with liver fibrosis. Mol Med 2008, 14:365-373

12. Raskopf E, Vogt A, Sauerbruch T, Schmitz V: siRNA targeting VEGF inhibits hepatocellular carcinoma growth and tumor angiogenesis in vivo. J Hepatol 2008, 49:977-984

13. Yan X, Chua MS, He J, So SK: Small interfering RNA targeting CDC25B inhibits liver tumor growth in vitro and in vivo. Mol Cancer 2008, 7:1-9

14. Sass G, Leukel P, Schmitz V, Raskopf E, Ocker M, Neureiter D, Meissnitzer M, Tasika E, Tannapfel A, Tiegs G: Inhibition of heme oxygenase 1 expression by small interfering RNA decreases orthotopic tumor growth in livers of mice. Int $J$ Cancer 2008, 123:1269-1277

15. Kota J, Chivukula RR, O'Donnell KA, Wentzel EA, Montgomery CL, Hwang HW, Chang TC, Vivekanandan P, Torbenson M, Clark KR, Mendell JR, Mendell JT: Therapeutic microRNA delivery suppresses tumorigenesis in a murine liver cancer model. Cell 2009, 137:1005-1017

16. Fernandez M, Semela D, Bruix J, Colle I, Pinzani M, Bosch J: Angiogenesis in liver disease. J Hepatol 2009, 50:604-620

17. Nascimento C, Bottino A, Nogueira C, Pannain V: Analysis of morphological variables and arterialization in the differential diagnosis of hepatic nodules in explanted cirrhotic livers. Diagn Pathol 2007, 2:51

18. Yang ZF, Poon RT: Vascular changes in hepatocellular carcinoma. Anat Rec (Hoboken) 2008, 291:721-734

19. Kaseb AO, Hanbali A, Cotant M, Hassan MM, Wollner I, Philip PA: Vascular endothelial growth factor in the management of hepatocellular carcinoma: a review of literature. Cancer 2009, 115:4895-4906

20. Dupuy E, Hainaud P, Villemain A, Bodevin-Phedre E, Brouland JP, Briand P, Tobelem G: Tumoral angiogenesis and tissue factor expression during hepatocellular carcinoma progression in a transgenic mouse model. J Hepatol 2003, 38:793-802

21. Hainaud P, Contreres JO, Villemain A, Liu LX, Plouet J, Tobelem G Dupuy E: The role of the vascular endothelial growth factor-Delta-like 4 ligand/Notch4-ephrin B2 cascade in tumor vessel remodeling and endothelial cell functions. Cancer Res 2006, 66:8501-8510

22. Bonnin P, Villemain A, Vincent F, Debbabi H, Silvestre JS, Contreres JO, Levy BI, Tobelem G, Dupuy E: Ultrasonic assessment of hepatic blood flow as a marker of mouse hepatocarcinoma. Ultrasound Med Biol 2007, 33:561-570

23. Kleinman ME, Yamada K, Takeda A, Chandrasekaran V, Nozaki M Baffi JZ, Albuquerque RJ, Yamasaki S, Itaya M, Pan Y, Appukuttan B, Gibbs D, Yang Z, Kariko K, Ambati BK, Wilgus TA, DiPietro LA, Sakurai E, Zhang K, Smith JR, Taylor EW, Ambati J: Sequence- and target-independent angiogenesis suppression by siRNA via TLR3. Nature 2008, 452:591-597

24. Cho WG, Albuquerque RJ, Kleinman ME, Tarallo V, Greco A, Nozaki M, Green MG, Baffi JZ, Ambati BK, De Falco M, Alexander JS, Brunetti A, De Falco S, Ambati J: Small interfering RNA-induced TLR3 activation inhibits blood and lymphatic vessel growth. Proc Natl Acad Sci USA 2009, 106:7137-7142

25. Dubois N, Bennoun M, Allemand I, Molina T, Grimber G, Daudet- 
Monsac M, Abelanet R, Briand P: Time-course development of differentiated hepatocarcinoma and lung metastasis in transgenic mice. J Hepatol 1991, 13:227-239

26. Ding S, Merkulova-Rainon T, Han ZC, Tobelem G: HGF receptor up-regulation contributes to the angiogenic phenotype of human endothelial cells and promotes angiogenesis in vitro. Blood 2003, 101:4816-4822

27. Sulpice E, Plouet J, Berge M, Allanic D, Tobelem G, MerkulovaRainon T: Neuropilin-1 and neuropilin-2 act as coreceptors, potentiating proangiogenic activity. Blood 2008, 111:2036-2045

28. Siegel AB, Cohen El, Ocean A, Lehrer D, Goldenberg A, Knox JJ, Chen H, Clark-Garvey S, Weinberg A, Mandeli J, Christos P, Mazumdar M, Popa E, Brown RS Jr, Rafii S, Schwartz JD: Phase II trial evaluating the clinical and biologic effects of bevacizumab in unresectable hepatocellular carcinoma. J Clin Oncol 2008, 26:2992-2998

29. Llovet JM, Ricci S, Mazzaferro V, Hilgard P, Gane E, Blanc JF, de Oliveira AC, Santoro A, Raoul JL, Forner A, Schwartz M, Porta C, Zeuzem S, Bolondi L, Greten TF, Galle PR, Seitz JF, Borbath I, Haussinger D, Giannaris T, Shan M, Moscovici M, Voliotis D, Bruix J: Sorafenib in advanced hepatocellular carcinoma. N Engl J Med 2008, 359:378-390

30. Arbuthnot P, Ely A, Weinberg MS: Hepatic delivery of RNA interference activators for therapeutic application. Curr Gene Ther 2009, 9:91-103

31. Jackson AL, Linsley PS: Recognizing and avoiding siRNA off-target effects for target identification and therapeutic application. Nat Rev Drug Discov 2010, 9:57-67

32. Seki E, Brenner DA: Toll-like receptors and adaptor molecules in liver disease: update. Hepatology 2008, 48:322-335

33. Vercammen E, Staal J, Beyaert R: Sensing of viral infection and activation of innate immunity by toll-like receptor 3 . Clin Microbiol Rev 2008, 21:13-25

34. Wu J, Lu M, Meng Z, Trippler M, Broering R, Szczeponek A, Krux F, Dittmer U, Roggendorf M, Gerken G, Schlaak JF: Toll-like receptormediated control of HBV replication by nonparenchymal liver cells in mice. Hepatology 2007, 46:1769-1778

35. Yoneda K, Sugimoto K, Shiraki K, Tanaka J, Beppu T, Fuke H,
Yamamoto N, Masuya M, Horie R, Uchida K, Takei Y: Dual topology of functional Toll-like receptor 3 expression in human hepatocellular carcinoma: differential signaling mechanisms of TLR3-induced NFkappaB activation and apoptosis. Int J Oncol 2008, 33:929-936

36. Chin Al, Miyahira AK, Covarrubias A, Teague J, Guo B, Dempsey PW Cheng G: Toll-like receptor 3-mediated suppression of TRAMP prostate cancer shows the critical role of type I interferons in tumor immune surveillance. Cancer Res 2010, 70:2595-2603

37. Meadows SK, Eriksson M, Barber A, Sentman CL: Human NK cell IFN-gamma production is regulated by endogenous TGF-beta. Int Immunopharmacol 2006, 6:1020-1028

38. Ghosh TK, Mickelson DJ, Solberg JC, Lipson KE, Inglefield JR, Alkan SS: TLR-TLR cross talk in human PBMC resulting in synergistic and antagonistic regulation of type- 1 and 2 interferons. IL-12 and TNFalpha. Int Immunopharmacol 2007, 7:1111-1121

39. Friesel R, Komoriya A, Maciag T: Inhibition of endothelial cell proliferation by gamma-interferon. J Cell Biol 1987, 104:689-696

40. Sidky YA, Borden EC: Inhibition of angiogenesis by interferons: effects on tumor- and lymphocyte-induced vascular responses. Cancer Res 1987, 47:5155-5161

41. Airoldi I, Meazza R, Croce M, Di CE, Piazza T, Cocco C, D'Antuono T, Pistoia V, Ferrini S, Corrias MV: Low-dose interferon-gamma-producing human neuroblastoma cells show reduced proliferation and delayed tumorigenicity. Br J Cancer 2004, 90:2210-2218

42. Ribatti D, Nico B, Pezzolo A, Vacca A, Meazza R, Cinti R, Carlini B, Parodi F, Pistoia V, Corrias MV: Angiogenesis in a human neuroblastoma xenograft model: mechanisms and inhibition by tumour-derived interferon-gamma. Br J Cancer 2006, 94:1845-1852

43. Fischer S, Nishio M, Peters SC, Tschernatsch M, Walberer M, Weidemann S, Heidenreich R, Couraud PO, Weksler BB, Romero IA, Gerriets T, Preissner KT: Signaling mechanism of extracellular RNA in endothelial cells. FASEB J 2009, 23:2100-2109

44. Shibamiya A, Hersemeyer K, Schmidt WT, Sedding D, Daniel JM, Bauer S, Koyama T, Preissner KT, Kanse SM: A key role for Toll-like receptor-3 in disrupting the hemostasis balance on endothelial cells. Blood 2009, 113:714-722 